Kragujevac Journal of Mathematics

Volume 42(1) (2018), Pages 15-27.

\title{
NEW INEQUALITIES ON HERMITE-HADAMARD UTILIZING FRACTIONAL INTEGRALS
}

\author{
SHAHID QAISAR ${ }^{1}$, MUHAMMAD IQBAL $^{2,3}$, SABIR HUSSAIN $^{4}$, SAAD I. BUTT $^{5}$, \\ AND MUHAMMAD A. MERAJ ${ }^{1}$
}

\begin{abstract}
In the present article, firstly authors have established an integral identity for Riemann-Liouville fractional integrals. Secondly, some Hermite-Hadamard type integral inequalities utilizing this integral identity are obtained and presented results have some closely connection with $[$ M. Z. Sarikaya, N. Aktan, On the generalization of some integral inequalities and their applications, Mathematical and Computer Modeling 54(9) (2011), 2175-2182].
\end{abstract}

\section{INTRODUCTION}

The usefulness of inequalities including convex functions is recognized from the soonest beginning stage and is now widely acknowledged as one of the prime driving forces behind the development of several modern branches of mathematics and has been given considerable attention. One of the most famous inequalities for convex functions is Hermite-Hadamard's inequality, stated as [7].

Let $f: I \subset \mathbb{R} \rightarrow \mathbb{R}$ be a convex function on the interval $I$ of real numbers and $a, b \in I$ with $a<b$. Then

$$
f\left(\frac{a+b}{2}\right) \leq \frac{1}{b-a} \int_{a}^{b} f(x) d x \leq \frac{f(a)+f(b)}{2} .
$$

Both inequalities hold in the reversed direction for $f$ to be concave.

Some key definitions and mathematical preliminaries of fractional calculus theory which are used further as a piece of this paper.

\footnotetext{
Key words and phrases. Hermite-Hadamard's inequality, Riemann-Liouville fractional integration, convex functions, power-mean inequality.

2010 Mathematics Subject Classification. Primary: 26D07, 26D10. Secondary: 26A15, 26A51.

Received: May 13, 2016.

Accepted: November 11, 2016.
} 
Definition 1.1. Let $f \in L^{1}[a, b]$. The left-sided and right-sided Riemann-Liouville fractional integrals of order $\alpha>0$ with $a \geq 0$ are defined by

$$
J_{a^{+}}^{\alpha} f(x)=\frac{1}{\Gamma(\alpha)} \int_{a}^{x}(x-t)^{\alpha-1} f(t) d t, \quad a<x
$$

and

$$
J_{b^{-}}^{\alpha} f(x)=\frac{1}{\Gamma(\alpha)} \int_{x}^{b}(t-x)^{\alpha-1} f(t) d t, \quad x<b
$$

respectively, where $\Gamma(\cdot)$ is Gamma function and its definition is $\Gamma(\alpha)=\int_{0}^{\infty} e^{-u} u^{\alpha-1} d u$. It is to be noted that $J_{a^{+}}^{0} f(x)=J_{b^{-}}^{0} f(x)=f(x)$.

In the case of $\alpha=1$, the fractional integral reduces to the classical integral.

Properties relating to this operator can be found in [9] and for useful details on Hermite-Hadamard type inequalities connected with fractional integral inequalities, readers are directed to $[1-6,8,11,14]$.

In [14] Sarikaya et al. proved a variant of Hermite-Hadamard's inequalities for fractional integral which follows as.

Theorem 1.1. Let $f:[a, b] \rightarrow \mathbb{R}$ be a positive function with $0 \leq a<b$ and $f \in L^{1}[a, b]$. If $f$ is convex function on $[a, b]$, then the following inequalities for fractional integrals hold

$$
f\left(\frac{a+b}{2}\right) \leq \frac{\Gamma(\alpha+1)}{2(b-a)^{\alpha}}\left[J_{a^{+}}^{\alpha} f(b)+J_{b^{-}}^{\alpha} f(a)\right] \leq \frac{f(a)+f(b)}{2},
$$

with $\alpha>0$.

Remark 1.1. For $\alpha=1$, inequality (1.2) reduces to inequality (1.1).

In [15] Sarikaya et al. proved some inequalities related to Hermite-Hadamard's inequalities for functions whose derivatives in absolute value at certain powers are convex functions as follows.

Theorem 1.2. Let $I \subset \mathbb{R}$ be an open interval, $a, b \in I$ with $a<b$ and $f:[a, b] \rightarrow \mathbb{R}$ be a twice differentiable function such that $f^{\prime \prime}$ is integrable and $0<\lambda \leq 1$ on $(a, b)$ with $a<b$. If $\left|f^{\prime \prime}\right|$ is a convex on $[a, b]$, then the following inequality holds

$$
\begin{aligned}
& \left|(\lambda-1) f\left(\frac{a+b}{2}\right)-\lambda \frac{f(a)+f(b)}{2}+\frac{1}{b-a} \int_{a}^{b} f(x) d x\right| \\
& \leq \begin{cases}\frac{(b-a)^{2}}{12}\left[\left(\lambda^{4}+(1+\lambda)(1-\lambda)^{3}+\frac{5 \lambda-3}{4}\right)\left|f^{\prime \prime}(a)\right|\right. & 0 \leq \lambda \leq 1 / 2, \\
\left.\quad+\left(\lambda^{4}+(2-\lambda) \lambda^{3}+\frac{1-3 \lambda}{4}\right)\left|f^{\prime \prime}(b)\right|\right], & 1 / 2 \leq \lambda \leq 1 . \\
\frac{(b-a)^{2}(3 \lambda-1)}{48}\left[\left|f^{\prime \prime}(a)\right|+\left|f^{\prime \prime}(b)\right|\right], & \end{cases}
\end{aligned}
$$


Proposition 1.1. Under the assumptions of Theorem 1.2 with $\lambda=0$, we get the inequality

$$
\left|\frac{1}{b-a} \int_{a}^{b} f(x) d x-f\left(\frac{a+b}{2}\right)\right| \leq \frac{(b-a)^{2}}{24}\left[\frac{\left|f^{\prime \prime}(a)\right|+\left|f^{\prime \prime}(b)\right|}{2}\right] .
$$

Theorem 1.3. Let $I \subset \mathbb{R}$ be an open interval, $a, b \in I$ with $a<b$ and $f:[a, b] \rightarrow \mathbb{R}$ be a twice differentiable function such that $f^{\prime \prime}$ is integrable and $0<\lambda \leq 1$ on $(a, b)$ with $a<b$. If $\left|f^{\prime \prime}\right|^{q}$ is a convex on $[a, b], q \geq 1$ then the following inequality holds

$$
\begin{aligned}
& \left|(\lambda-1) f\left(\frac{a+b}{2}\right)-\lambda \frac{f(a)+f(b)}{2}+\frac{1}{b-a} \int_{a}^{b} f(x) d x\right|
\end{aligned}
$$

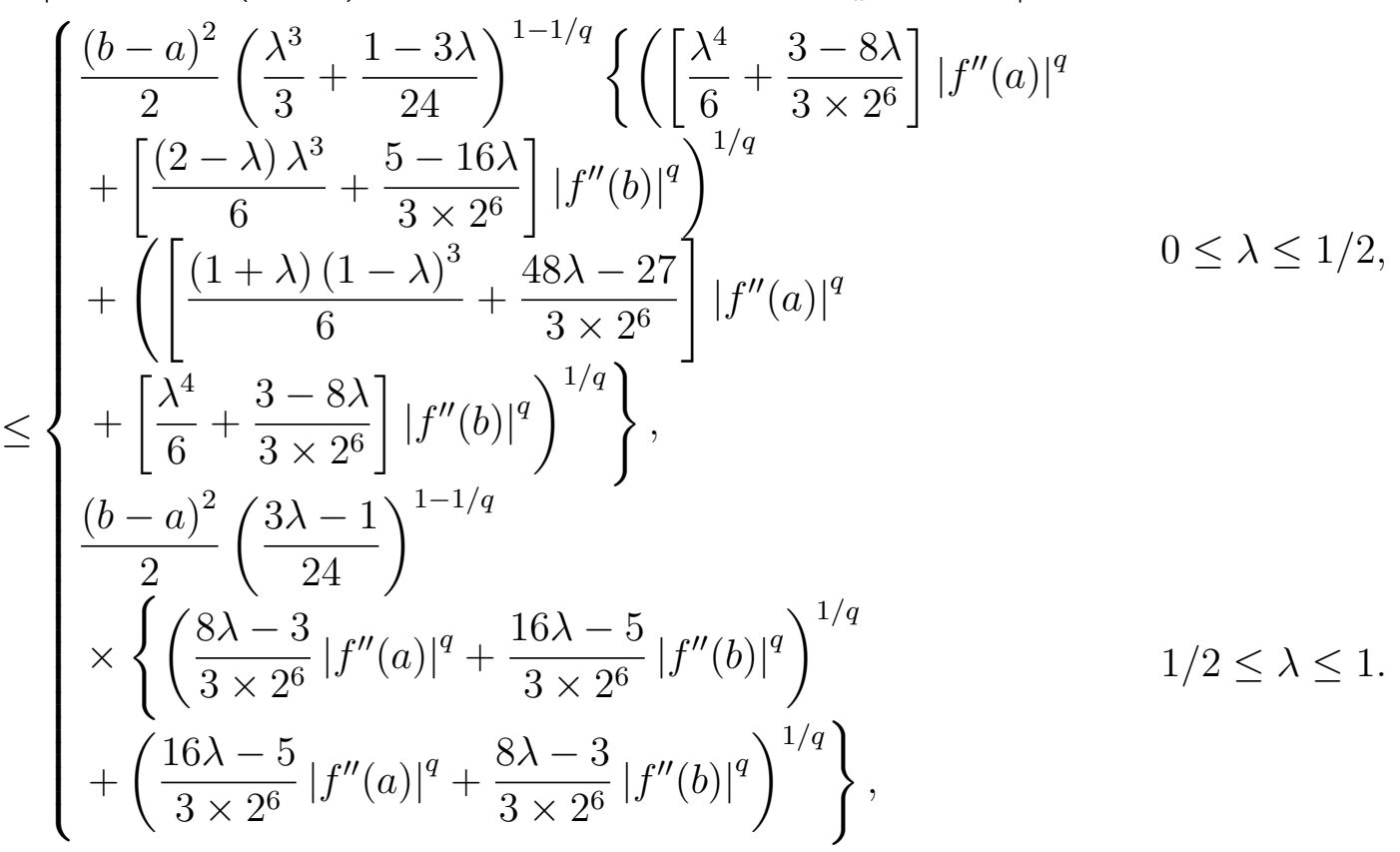

Proposition 1.2. Under the assumptions of Theorem 1.3 with $\lambda=0$, then we get the "midpoint inequality"

$$
\begin{aligned}
& \left|\frac{1}{b-a} \int_{a}^{b} f(x) d x-f\left(\frac{a+b}{2}\right)\right| \\
\leq & \frac{(b-a)^{2}}{48}\left[\left(\frac{5\left|f^{\prime \prime}(a)\right|^{q}+3\left|f^{\prime \prime}(b)\right|^{q}}{8}\right)^{1 / q}+\left(\frac{3\left|f^{\prime \prime}(a)\right|^{q}+5\left|f^{\prime \prime}(a)\right|^{q}}{8}\right)^{1 / q}\right] .
\end{aligned}
$$

The aim of this paper is to provide a unified approach to establish HermiteHadamard type inequalities for Riemann-Liouville fractional integral using the convexity as well as concavity, for functions whose absolute values of second derivatives are convex. we will derive a general integral identity for convex functions.

\section{Main Results}

In order to prove our main results we need the following integral identity. 
Lemma 2.1. Let $I \subset \mathbb{R}$ be an open interval, $a, b \in I$ with $a<b$ and $f:[a, b] \rightarrow \mathbb{R}$ be a twice differentiable function such that $f^{\prime \prime}$ is integrable and $0<\alpha \leq 1$ on $(a, b)$ with $a<b$. If $\left|f^{\prime \prime}\right|$ is a convex on $[a, b]$, then the following identity for Riemann-Liouville fractional integrals holds

$$
\frac{\Gamma(\alpha+1)}{2(b-a)^{\alpha}}\left[J_{a^{+}}^{\alpha} f(b)+J_{b^{-}}^{\alpha} f(a)\right]-f\left(\frac{a+b}{2}\right)=\frac{(b-a)^{2}}{2^{\alpha+3}(\alpha+1)} \sum_{k=1}^{4} I_{k},
$$

where

$$
\begin{aligned}
& I_{1}=\int_{0}^{1}(1-t)^{\alpha+1} f^{\prime \prime}\left(t a+(1-t) \frac{a+b}{2}\right) d t \\
& I_{2}=\int_{0}^{1}\left((1+t)^{\alpha+1}-2^{\alpha}(1+t)+\alpha 2^{\alpha}(1-t)\right) f^{\prime \prime}\left(t b+(1-t) \frac{a+b}{2}\right) d t \\
& I_{3}=\int_{0}^{1}(1-t)^{\alpha+1} f^{\prime \prime}\left(t b+(1-t) \frac{a+b}{2}\right) d t \\
& I_{4}=\int_{0}^{1}\left((1+t)^{\alpha+1}-2^{\alpha}(1+t)+\alpha 2^{\alpha}(1-t)\right) f^{\prime \prime}\left(t a+(1-t) \frac{a+b}{2}\right) d t .
\end{aligned}
$$

Proof. Integrating by parts

$$
\begin{aligned}
I_{1}= & \int_{0}^{1}(1-t)^{\alpha+1} f^{\prime \prime}\left(t a+(1-t) \frac{a+b}{2}\right) d t \\
= & \left.\frac{2(1-t)^{\alpha+1} f^{\prime}\left(t a+(1-t) \frac{a+b}{2}\right) d t}{a-b}\right|_{0} ^{1} \\
& +\frac{2(\alpha+1)}{a-b} \int_{0}^{1}(1-t)^{\alpha} f^{\prime}\left(t a+(1-t) \frac{a+b}{2}\right) d t \\
= & \frac{2}{b-a} f^{\prime}\left(\frac{a+b}{2}\right)+\frac{2(\alpha+1)}{a-b}\left[\left.\frac{2(1-t)^{\alpha} f^{\prime}\left(t a+(1-t) \frac{a+b}{2}\right) d t}{a-b}\right|_{0} ^{1}\right. \\
& \left.+\frac{2 \alpha}{a-b} \int_{0}^{1}(1-t)^{\alpha} f\left(t a+(1-t) \frac{a+b}{2}\right) d t\right] \\
= & \frac{2}{b-a} f^{\prime}\left(\frac{a+b}{2}\right)+\frac{2(\alpha+1)}{a-b}\left[\frac{-2}{a-b} f\left(\frac{a+b}{2}\right)\right. \\
& \left.-\frac{2 \alpha}{b-a} \int_{0}^{1}(1-t)^{\alpha-1} f\left(t a+(1-t) \frac{a+b}{2}\right) d t\right] \\
= & \frac{2}{b-a} f^{\prime}\left(\frac{a+b}{2}\right)-\frac{4(\alpha+1)}{(b-a)^{2}} f\left(\frac{a+b}{2}\right)+\frac{\alpha(\alpha+1) 2^{\alpha+2}}{(b-a)^{\alpha}} J_{1}, \\
I_{2}= & \int_{0}^{1}\left((1+t)^{\alpha+1}-2^{\alpha}(1+t)+\alpha 2^{\alpha}(1-t)\right) f^{\prime \prime}\left(t b+(1-t) \frac{a+b}{2}\right) d t
\end{aligned}
$$


NEW INEQUALITIES ON HERMITE-HADAMARD UTILIZING FRACTIONAL INTEGRALS

$$
\begin{aligned}
= & \left.\frac{\left.2\left[(1+t)^{\alpha+1}-2^{\alpha}(1+t)+\alpha 2^{\alpha}(1-t)\right] f^{\prime}\left(t b+(1-t) \frac{a+b}{2}\right) d t\right)}{b-a}\right|_{0} ^{1} \\
& +\frac{2(\alpha+1)}{b-a} \int_{0}^{1}\left[(\alpha+1)(1+t)^{\alpha}-2^{\alpha}-\alpha 2^{\alpha}\right] f^{\prime}\left(t b+(1-t) \frac{a+b}{2}\right) d t \\
= & -\frac{2}{b-a}\left[1+2^{\alpha}(\alpha-1)\right] f^{\prime}\left(\frac{a+b}{2}\right) \\
& +\frac{2(\alpha+1)}{b-a} \int_{0}^{1}\left[(\alpha+1)(1+t)^{\alpha}-2^{\alpha}-\alpha 2^{\alpha}\right] f^{\prime}\left(t b+(1-t) \frac{a+b}{2}\right) d t \\
= & -\frac{2}{b-a}\left[1+2^{\alpha}(\alpha-1)\right] f^{\prime}\left(\frac{a+b}{2}\right) \\
& +\frac{2(\alpha+1)}{b-a}\left[\left.\frac{\left.2\left[(\alpha+1)(1+t)^{\alpha}-2^{\alpha}-\alpha 2^{\alpha}\right] f^{\prime}\left(t b+(1-t) \frac{a+b}{2}\right) d t\right)}{b-a}\right|_{0} ^{1}\right. \\
& \left.-\frac{2 \alpha(\alpha+1)}{b-a} \int_{0}^{1}(1+t)^{\alpha-1} f\left(t b+(1-t) \frac{a+b}{2}\right) d t\right] \\
= & -\frac{2}{b-a}\left[1+2^{\alpha}(\alpha-1)\right] f^{\prime}\left(\frac{a+b}{2}\right)+\frac{4(\alpha+1)}{(b-a)^{2}}\left(2^{\alpha}-1\right) f\left(\frac{a+b}{2}\right) \\
& -\frac{\alpha(\alpha+1) 2^{\alpha+2}}{(b-a)^{\alpha}} J_{2} .
\end{aligned}
$$

Analogously:

$$
\begin{aligned}
I_{3}= & -\frac{2}{b-a} f^{\prime}\left(\frac{a+b}{2}\right)-\frac{4(\alpha+1)}{(b-a)^{2}} f\left(\frac{a+b}{2}\right)+\frac{\alpha(\alpha+1) 2^{\alpha+2}}{(b-a)^{\alpha}} J_{3}, \\
I_{4}= & -\frac{2}{b-a}\left[2^{\alpha}(1-\alpha)-1\right] f^{\prime}\left(\frac{a+b}{2}\right)-\frac{4(\alpha+1)}{(b-a)^{2}}\left(2^{\alpha}-1\right) f\left(\frac{a+b}{2}\right) \\
& +\frac{\alpha(\alpha+1) 2^{\alpha+2}}{(b-a)^{\alpha}} J_{4} .
\end{aligned}
$$

Adding above equalities, we get

$$
\frac{2}{b-a} f\left(\frac{a+b}{2}\right)-\frac{\alpha}{(b-a)^{\alpha}}\left[J_{1}+J_{2}+J_{3}+J_{4}\right]=I_{1}+I_{2}+I_{3}+I_{4} .
$$

Now making suitable substitutions, we have

$$
\begin{aligned}
J_{1} & =\int_{0}^{1}(1-t)^{\alpha+1} f^{\prime \prime}\left(t a+(1-t) \frac{a+b}{2}\right) d t \\
& =\frac{2^{\alpha}}{(b-a)^{\alpha}} \int_{a}^{a+b / 2}(u-a)^{\alpha-1} f(u) d u, \\
J_{2} & =\int_{0}^{1}(1+t)^{\alpha+1} f^{\prime \prime}\left(t b+(1-t) \frac{a+b}{2}\right) d t
\end{aligned}
$$




$$
\begin{aligned}
& =\frac{2^{\alpha}}{(b-a)^{\alpha}} \int_{a+b / 2}^{b}(u-a)^{\alpha-1} f(u) d u \\
J_{1}+J_{2} & =\frac{2^{\alpha}}{(b-a)^{\alpha}} \int_{a}^{b}(u-a)^{\alpha-1} f(u) d u=\frac{2^{\alpha} \Gamma(\alpha)}{(b-a)^{\alpha}} J_{b^{-}}^{\alpha} f(a),
\end{aligned}
$$

likewise

$$
\begin{aligned}
J_{3} & =\int_{0}^{1}(1-t)^{\alpha+1} f^{\prime \prime}\left(t b+(1-t) \frac{a+b}{2}\right) d t \\
& =\frac{2^{\alpha}}{(b-a)^{\alpha}} \int_{a+b / 2}^{b}(b-u)^{\alpha-1} f(u) d u \\
J_{4} & =\int_{0}^{1}(1+t)^{\alpha+1} f^{\prime \prime}\left(t a+(1-t) \frac{a+b}{2}\right) d t \\
& =\frac{2^{\alpha}}{(b-a)^{\alpha}} \int_{a}^{a+b / 2}(b-u)^{\alpha-1} f(u) d u, \\
J_{3}+J_{4} & =\frac{2^{\alpha}}{(b-a)^{\alpha}} \int_{a}^{b}(b-u)^{\alpha-1} f(u) d u=\frac{2^{\alpha} \Gamma(\alpha)}{(b-a)^{\alpha}} J_{a^{+}}^{\alpha} f(b),
\end{aligned}
$$

which completes our proof.

Theorem 2.1. Let $I \subset \mathbb{R}$ be an open interval, $a, b \in I$ with $a<b$ and $f:[a, b] \rightarrow \mathbb{R}$ be a twice differentiable function such that $f^{\prime \prime}$ is integrable and $0<\alpha \leq 1$ on $(a, b)$ with $a<b$. If $\left|f^{\prime \prime}\right|$ is a convex on $[a, b]$, then the following identity for Riemann-Liouville fractional integrals holds

$$
\begin{aligned}
& \left|\frac{\Gamma(\alpha+1)}{2(b-a)^{\alpha}}\left[J_{a^{+}}^{\alpha} f(b)+J_{b^{-}}^{\alpha} f(a)\right]-f\left(\frac{a+b}{2}\right)\right| \\
\leq & \frac{(b-a)^{2}}{2^{\alpha+4}(\alpha+1)}\left(K_{1}+K_{2}+K_{3}+K_{4}\right)\left(\left|f^{\prime \prime}(a)\right|+\left|f^{\prime \prime}(b)\right|\right) .
\end{aligned}
$$

Proof. By using the properties of modulus on Lemma 2.1, we have

$$
\left|\frac{\Gamma(\alpha+1)}{2(b-a)^{\alpha}}\left[J_{a^{+}}^{\alpha} f(b)+J_{b^{-}}^{\alpha} f(a)\right]-f\left(\frac{a+b}{2}\right)\right| \leq \frac{(b-a)^{2}}{2^{\alpha+3}(\alpha+1)} \sum_{k=1}^{4}\left|I_{k}\right| .
$$

Now, using convexity of $\left|f^{\prime \prime}\right|$, we have

$$
\begin{aligned}
\left|I_{1}\right| & \leq \int_{0}^{1}(1-t)^{\alpha+1}\left|f^{\prime \prime}\left(t a+(1-t) \frac{a+b}{2}\right)\right| d t \\
& =\int_{0}^{1}(1-t)^{\alpha+1}\left|f^{\prime \prime}\left(\frac{1+t}{2} a+\frac{1-t}{2} b\right)\right| d t \\
& \leq \int_{0}^{1}(1-t)^{\alpha+1}\left\{\left(\frac{1+t}{2}\right)\left|f^{\prime \prime}(a)\right|+\left(\frac{1-t}{2}\right)\left|f^{\prime \prime}(b)\right|\right\} d t
\end{aligned}
$$




$$
=\frac{K_{1}}{2}\left|f^{\prime \prime}(a)\right|+\frac{K_{2}}{2}\left|f^{\prime \prime}(b)\right|
$$

Analogously:

$$
\left|I_{3}\right| \leq \frac{K_{1}}{2}\left|f^{\prime \prime}(b)\right|+\frac{K_{2}}{2}\left|f^{\prime \prime}(a)\right|
$$

By using the convexity on $\left|f^{\prime \prime}\right|$ and fact that for $\alpha \in(0,1]$ and for all $t \in[0,1]$,

$$
\begin{aligned}
\left|I_{2}\right| \leq & \int_{0}^{1}\left((1+t)^{\alpha+1}-2^{\alpha}(1+t)+\alpha 2^{\alpha}(1-t)\right)\left|f^{\prime \prime}\left(t a+(1-t) \frac{a+b}{2}\right)\right| d t \\
= & \int_{0}^{1}\left((1+t)^{\alpha+1}-2^{\alpha}(1+t)+\alpha 2^{\alpha}(1-t)\right)\left|f^{\prime \prime}\left(\frac{1+t}{2} b+\frac{1-t}{2} a\right)\right| d t \\
\leq & \int_{0}^{1}\left\{(1+t)^{\alpha+1}-2^{\alpha}(1+t)+\alpha 2^{\alpha}(1-t)\right\} \\
& \times\left\{\left(\frac{1+t}{2}\right)\left|f^{\prime \prime}(b)\right|+\left(\frac{1-t}{2}\right)\left|f^{\prime \prime}(a)\right|\right\} d t \\
= & \frac{K_{3}}{2}\left|f^{\prime \prime}(b)\right|+\frac{K_{4}}{2}\left|f^{\prime \prime}(a)\right| .
\end{aligned}
$$

Similarly

$$
\left|I_{4}\right| \leq \frac{K_{3}}{2}\left|f^{\prime \prime}(a)\right|+\frac{K_{4}}{2}\left|f^{\prime \prime}(b)\right|
$$

To get desired result, adding above inequalities and it is very easy to check

$$
\begin{aligned}
K_{1} & =\int_{0}^{1}(1-t)^{\alpha+1}(1+t) d t=\frac{1}{\alpha+2}+\frac{1}{(\alpha+2)(\alpha+3)}, \\
K_{2} & =\int_{0}^{1}(1-t)^{\alpha+2} d t=\frac{1}{\alpha+3}, \\
K_{3} & =\int_{0}^{1}\left\{(1+t)^{\alpha+1}-2^{\alpha}(1+t)+\alpha 2^{\alpha}(1-t)\right\}(1+t) d t \\
& =\frac{2^{\alpha+3}}{\alpha+3}-\frac{1}{\alpha+3}-\frac{2^{\alpha+3}}{3}+\alpha \frac{2^{\alpha+1}}{3}+\frac{2^{\alpha}}{3}, \\
K_{4} & =\int_{0}^{1}\left\{(1+t)^{\alpha+1}-2^{\alpha}(1+t)+\alpha 2^{\alpha}(1-t)\right\}(1-t) d t \\
& =-\frac{1}{\alpha+2}+\frac{2^{\alpha+3}}{(\alpha+2)(\alpha+3)}-\frac{1}{(\alpha+2)(\alpha+3)}-\frac{2^{\alpha+1}}{3}+\alpha \frac{2^{\alpha}}{3},
\end{aligned}
$$

which completes the proof.

Remark 2.1. If we take $\alpha=1$ in Theorem 2.1 then inequality (2.1) reduces to inequality

$$
\left|\frac{1}{b-a} \int_{a}^{b} f(x) d x-f\left(\frac{a+b}{2}\right)\right| \leq \frac{(b-a)^{2}}{24}\left[\frac{\left|f^{\prime \prime}(a)\right|+\left|f^{\prime \prime}(b)\right|}{2}\right] .
$$


Theorem 2.2. Let $I \subset \mathbb{R}$ be an open interval, $a, b \in I$ with $a<b$ and $f:[a, b] \rightarrow \mathbb{R}$ be a twice differentiable function such that $f^{\prime \prime}$ is integrable and $0<\alpha \leq 1$ on $(a, b)$ with $a<b$. If $\left|f^{\prime \prime}\right|$ is a convex on $[a, b]$, then the following identity for Riemann-Liouville fractional integrals holds

$$
\begin{aligned}
& \left|\frac{\Gamma(\alpha+1)}{2(b-a)^{\alpha}}\left[J_{a^{+}}^{\alpha} f(b)+J_{b^{-}}^{\alpha} f(a)\right]-f\left(\frac{a+b}{2}\right)\right| \\
\leq & \frac{(b-a)^{2}}{2^{\alpha+3}(\alpha+1)}\left[\left(K_{5}+K_{6}\right)\left\{\left|f^{\prime \prime}(a)\right|+\left|f^{\prime \prime}(b)\right|\right\}+2\left(K_{2}+K_{4}\right)\left|f^{\prime \prime}\left(\frac{a+b}{2}\right)\right|\right] .
\end{aligned}
$$

Proof. By using the properties of modulus on Lemma 2.1, we have

$$
\left|\frac{\Gamma(\alpha+1)}{2(b-a)^{\alpha}}\left[J_{a^{+}}^{\alpha} f(b)+J_{b^{-}}^{\alpha} f(a)\right]-f\left(\frac{a+b}{2}\right)\right| \leq \frac{(b-a)^{2}}{2^{\alpha+3}(\alpha+1)} \sum_{k=1}^{4}\left|I_{k}\right| .
$$

Now, using convexity of $\left|f^{\prime \prime}\right|$, we have

$$
\begin{aligned}
\left|I_{1}\right| & \leq \int_{0}^{1}(1-t)^{\alpha+1}\left|f^{\prime \prime}\left(t a+(1-t) \frac{a+b}{2}\right)\right| d t \\
& \leq \int_{0}^{1}(1-t)^{\alpha+1}\left\{t\left|f^{\prime \prime}(a)\right|+(1-t)\left|f^{\prime \prime}\left(\frac{a+b}{2}\right)\right|\right\} d t \\
& =\frac{K_{5}}{2}\left|f^{\prime \prime}(a)\right|+\frac{K_{2}}{2}\left|f^{\prime \prime}\left(\frac{a+b}{2}\right)\right| .
\end{aligned}
$$

Analogously

$$
\left|I_{3}\right| \leq \frac{K_{5}}{2}\left|f^{\prime \prime}(b)\right|+\frac{K_{2}}{2}\left|f^{\prime \prime}\left(\frac{a+b}{2}\right)\right| .
$$

By using the convexity on $\left|f^{\prime \prime}\right|$ and fact that for $\alpha \in(0,1]$ and for all $t \in[0,1]$,

$$
\begin{aligned}
\left|I_{2}\right| & \leq \int_{0}^{1}\left((1+t)^{\alpha+1}-2^{\alpha}(1+t)+\alpha 2^{\alpha}(1-t)\right)\left|f^{\prime \prime}\left(t a+(1-t) \frac{a+b}{2}\right)\right| d t \\
& =\int_{0}^{1}\left((1+t)^{\alpha+1}-2^{\alpha}(1+t)+\alpha 2^{\alpha}(1-t)\right)\left\{t\left|f^{\prime \prime}(a)\right|+(1-t)\left|f^{\prime \prime}\left(\frac{a+b}{2}\right)\right|\right\} d t \\
& =\frac{K_{6}}{2}\left|f^{\prime \prime}(b)\right|+\frac{K_{4}}{2}\left|f^{\prime \prime}\left(\frac{a+b}{2}\right)\right| .
\end{aligned}
$$

Similarly

$$
\begin{aligned}
\left|I_{4}\right| & \leq \frac{K_{7}}{2}\left|f^{\prime \prime}(a)\right|+\frac{K_{8}}{2}\left|f^{\prime \prime}\left(\frac{a+b}{2}\right)\right|, \\
& \left|\frac{\Gamma(\alpha+1)}{2(b-a)^{\alpha}}\left[J_{a^{+}}^{\alpha} f(b)+J_{b^{-}}^{\alpha} f(a)\right]-f\left(\frac{a+b}{2}\right)\right| \\
& \leq \frac{(b-a)^{2}}{2^{\alpha+3}(\alpha+1)}\left[\left(K_{5}+K_{6}\right)\left\{\left|f^{\prime \prime}(a)\right|+\left|f^{\prime \prime}(b)\right|\right\}+2\left(K_{2}+K_{4}\right)\left|f^{\prime \prime}\left(\frac{a+b}{2}\right)\right|\right] .
\end{aligned}
$$


It is very easy to check

$$
\begin{aligned}
K_{5} & =\int_{0}^{1} t(1-t)^{\alpha+1} d t=\frac{1}{(\alpha+2)(\alpha+3)} \\
K_{6} & =\int_{0}^{1}\left\{(1+t)^{\alpha+1}-2^{\alpha}(1+t)+\alpha 2^{\alpha}(1-t)\right\} t d t \\
& =\frac{2^{\alpha+2}}{\alpha+2}-\frac{2^{\alpha+3}}{(\alpha+2)(\alpha+3)}+\frac{1}{(\alpha+2)(\alpha+3)}+\alpha \frac{2^{\alpha}}{6}-\frac{5.2^{\alpha}}{6},
\end{aligned}
$$

which completes the proof.

Remark 2.2. If we take $\alpha=1$ in Theorem 2.2, then inequality (2.2) becomes as inequality

$$
\left|\frac{1}{b-a} \int_{a}^{b} f(x) d x-f\left(\frac{a+b}{2}\right)\right| \leq \frac{(b-a)^{2}}{48}\left[\left|f^{\prime \prime}(a)\right|+\left|f^{\prime \prime}(b)\right|\right] .
$$

The corresponding version for powers of the absolute value of the derivative is incorporated in the following theorem.

Theorem 2.3. Let $I \subset \mathbb{R}$ be an open interval, $a, b \in I$ with $a<b$ and $f:[a, b] \rightarrow \mathbb{R}$ be a twice differentiable function such that $f^{\prime \prime}$ is integrable and $0<\alpha \leq 1$ on $(a, b)$ with $a<b$. If $\left|f^{\prime \prime}\right|^{q}$ is a convex on $[a, b], q \geq 1$ then the following inequality holds

$$
\begin{aligned}
& \left|\frac{\Gamma(\alpha+1)}{2(b-a)^{\alpha}}\left[J_{a^{+}}^{\alpha} f(b)+J_{b^{-}}^{\alpha} f(a)\right]-f\left(\frac{a+b}{2}\right)\right| \\
\leq & \frac{(b-a)^{2}}{2^{\alpha+3}(\alpha+1)}\left[\left(K_{9}\right)^{1-1 / q}\left(\frac{K_{7}\left|f^{\prime \prime}(a)\right|^{q}+K_{2}\left|f^{\prime \prime}(b)\right|^{q}}{2}\right)^{1 / q}\right. \\
& +\left(\frac{K_{2}\left|f^{\prime \prime}(a)\right|^{q}+K_{7}\left|f^{\prime \prime}(b)\right|^{q}}{2}\right)^{1 / q}+\left(K_{10}\right)^{1-1 / q}\left(\frac{K_{4}\left|f^{\prime \prime}(a)\right|^{q}+K_{8}\left|f^{\prime \prime}(b)\right|^{q}}{2}\right)^{1 / q} \\
& \left.+\left(\frac{K_{8}\left|f^{\prime \prime}(a)\right|^{q}+K_{4}\left|f^{\prime \prime}(b)\right|^{q}}{2}\right)^{1 / q}\right]
\end{aligned}
$$

Proof. Using the well-known power-mean integral inequality for $q>1$, we have

$$
\left|I_{1}\right| \leq\left(\int_{0}^{1}(1-t)^{\alpha+1} d t\right)^{1-1 / q}\left(\int_{0}^{1}(1-t)^{\alpha+1}\left|f^{\prime \prime}\left(t a+(1-t) \frac{a+b}{2}\right)\right|^{q} d t\right)^{1 / q} .
$$

By the convexity of $\left|f^{\prime \prime}\right|^{q}$

$$
\left|I_{1}\right| \leq\left(K_{9}\right)^{1-1 / q}\left(K_{7} \frac{\left|f^{\prime \prime}(a)\right|^{q}}{2}+K_{2} \frac{\left|f^{\prime \prime}(b)\right|^{q}}{2}\right)^{1 / q}
$$

Analogously

$$
\left|I_{3}\right| \leq\left(K_{9}\right)^{1-1 / q}\left(K_{7} \frac{\left|f^{\prime \prime}(b)\right|^{q}}{2}+K_{2} \frac{\left|f^{\prime \prime}(a)\right|^{q}}{2}\right)^{1 / q}
$$




$$
\begin{aligned}
\left|I_{2}\right| \leq & \left(K_{10}\right)^{1-1 / q}\left(\int_{0}^{1}\left((1+t)^{\alpha+1}-2^{\alpha}(1+t)+\alpha 2^{\alpha}(1-t)\right)\right. \\
& \left.\times\left|f^{\prime \prime}\left(t b+(1-t) \frac{a+b}{2}\right)\right|^{q} d t\right)^{1 / q} .
\end{aligned}
$$

By the convexity of $\left|f^{\prime \prime}\right|^{q}$

$$
\left|I_{2}\right| \leq\left(K_{10}\right)^{1-1 / q}\left(K_{4} \frac{\left|f^{\prime \prime}(a)\right|^{q}}{2}+K_{8} \frac{\left|f^{\prime \prime}(b)\right|^{q}}{2}\right)^{1 / q}
$$

Analogously

$$
\left|I_{4}\right| \leq\left(K_{10}\right)^{1-1 / q}\left(K_{8} \frac{\left|f^{\prime \prime}(b)\right|^{q}}{2}+K_{4} \frac{\left|f^{\prime \prime}(a)\right|^{q}}{2}\right)^{1 / q} .
$$

It is very easy to check

$$
\begin{aligned}
K_{7} & =\int_{0}^{1} \frac{(1+t)}{2}(1-t)^{\alpha+1} d t=\frac{1}{\alpha+2}+\frac{1}{(\alpha+2)(\alpha+3)} \\
K_{8} & =\int_{0}^{1}\left\{(1+t)^{\alpha+1}-2^{\alpha}(1+t)+\alpha 2^{\alpha}(1-t)\right\}(1+t) d t \\
& =\frac{2^{\alpha+3}}{(\alpha+3)}-\frac{1}{(\alpha+3)}-\frac{7 \cdot 2^{\alpha}}{3}+\frac{\alpha 2^{\alpha+1}}{3} \\
K_{9} & =\int_{0}^{1}(1-t)^{\alpha+1} d t=\frac{1}{(\alpha+2)} \\
K_{10} & =\int_{0}^{1}\left((1+t)^{\alpha+1}-2^{\alpha}(1+t)+\alpha 2^{\alpha}(1-t)\right) d t \\
& =\frac{2^{\alpha+2}}{(\alpha+2)}-2^{\alpha+2}+2^{\alpha-1}-\frac{1}{\alpha+2}+\alpha 2^{\alpha-1} .
\end{aligned}
$$

Which completes the proof.

Remark 2.3. If we take $\alpha=1$ in Theorem 2.3, then inequality (2.3) reduces to inequality

$$
\begin{aligned}
& \left|\frac{1}{b-a} \int_{a}^{b} f(x) d x-f\left(\frac{a+b}{2}\right)\right| \\
\leq & \frac{(b-a)^{2}}{48}\left[\left(\frac{5\left|f^{\prime \prime}(a)\right|^{q}+3\left|f^{\prime \prime}(b)\right|^{q}}{8}\right)^{1 / q}+\left(\frac{3\left|f^{\prime \prime}(a)\right|^{q}+5\left|f^{\prime \prime}(a)\right|^{q}}{8}\right)^{1 / q}\right] .
\end{aligned}
$$

In the following, we obtain estimate of Hermite-Hadamard inequality (1.2) for concave functions.

Theorem 2.4. Let $f:[a, b] \rightarrow \mathbb{R}$ be a twice differentiable function on $(a, b)$ such that $f^{\prime 1}[a, b]$. If $\left|f^{\prime \prime}\right|^{q}$ is concave on $[a, b]$ for some fixed $p>1$ with $q=\frac{p}{p-1}$, then the 
following inequality for fractional integrals holds for $\alpha>0$

$$
\begin{aligned}
& \left|\frac{\Gamma(\alpha+1)}{2(b-a)^{\alpha}}\left[J_{a^{+}}^{\alpha} f(b)+J_{b^{-}}^{\alpha} f(a)\right]-f\left(\frac{a+b}{2}\right)\right| \\
\leq & \frac{(b-a)^{2}}{2^{\alpha+3}(\alpha+1)}\left[K _ { 9 } \left\{\left|f^{\prime \prime}\left((\alpha+2)\left\{\frac{K_{1} a+K_{2} b}{2}\right\}\right)\right|\right.\right. \\
& \left.+\left|f^{\prime \prime}\left((\alpha+2)\left\{\frac{K_{2} a+K_{1} b}{2}\right\}\right)\right|\right\} \\
& \left.+K_{10}\left|f^{\prime \prime}\left((\alpha+2)\left\{\frac{K_{3} a+K_{4} b}{2}\right\}\right)\right|+f^{\prime \prime}(\alpha+2)\left\{\frac{K_{4} a+K_{3} b}{2}\right\}\right] .
\end{aligned}
$$

Proof. Using the concavity of $\left|f^{\prime \prime}\right|^{q}$ and the power-mean inequality, we obtain

$$
\left|f^{\prime \prime}\right|^{q}>t\left|f^{\prime \prime}\right|^{q}+(1-t)\left|f^{\prime \prime}\right|^{q} \geq t\left|f^{\prime \prime}\right|^{q}+(1-t)\left|f^{\prime \prime}\right|^{q} .
$$

Hence

$$
\left|f^{\prime \prime}(t x+(1-t) y)\right| \geq t\left|f^{\prime \prime}(x)\right|+(1-t)\left|f^{\prime \prime}(y)\right|
$$

so, $\left|f^{\prime \prime}\right|$ is also concave. By the Jensen integral inequality, we have

$$
\begin{aligned}
\left|I_{1}\right| & \leq\left(\int_{0}^{1}(1-t)^{\alpha+1} d t\right)\left|f^{\prime \prime}\left(\frac{\int_{0}^{1}(1-t)^{\alpha+1}\left(\frac{1+t}{2} a+\frac{1-t}{2} b\right) d t}{\int_{0}^{1}(1-t)^{\alpha+1} d t}\right)\right|^{q} \\
& =K_{9}\left|f^{\prime \prime}\left((\alpha+2)\left\{\frac{K_{1} a+K_{2} b}{2}\right\}\right)\right|^{q} .
\end{aligned}
$$

Analogously

$$
\begin{aligned}
& \left|I_{2}\right| \leq K_{10}\left|f^{\prime \prime}\left((\alpha+2)\left\{\frac{K_{3} a+K_{4} b}{2}\right\}\right)\right|^{q}, \\
& \left|I_{3}\right| \leq K_{9}\left|f^{\prime \prime}\left((\alpha+2)\left\{\frac{K_{2} a+K_{1} b}{2}\right\}\right)\right|^{q}, \\
& \left|I_{4}\right| \leq K_{10}\left|f^{\prime \prime}\left((\alpha+2)\left\{\frac{K_{4} a+K_{3} b}{2}\right\}\right)\right|^{q},
\end{aligned}
$$

which completes the proof.

Corollary 2.1. If we take $\alpha=1$ in Theorem 2.4, then inequality (2.4) becomes

$$
\left|\frac{1}{b-a} \int_{a}^{b} f(x) d x-f\left(\frac{a+b}{2}\right)\right| \leq \frac{(b-a)^{2}}{48}\left[\left|f^{\prime \prime}\left(\frac{5 a+3 b}{8}\right)\right|+\left|f^{\prime \prime}\left(\frac{3 a+5 b}{8}\right)\right|\right] \text {. }
$$

Remark 2.4. The obtained Corollary 2.1 is an improvement of the inequality given as

$$
\left|\frac{1}{b-a} \int_{a}^{b} f(x) d x-f\left(\frac{a+b}{2}\right)\right| \leq \frac{(b-a)^{2}}{16}\left[\left|f^{\prime \prime}\left(\frac{3 a+b}{4}\right)\right|+\left|f^{\prime \prime}\left(\frac{a+3 b}{4}\right)\right|\right] .
$$


Acknowledgements. The authors are grateful to Dr S. M. Junaid Zaidi, Rector, COMSATS Institute of Information Technology, Pakistan for providing excellent research facilities. Funding The author S. Qaisar was partially supported by the Higher Education Commission of Pakistan [grant number No. 21-52/SRGP/R\&D/HEC/2014].

\title{
REFERENCES
}

[1] G. Anastassiou, M. R. Hooshmandasl, A. Ghasemi and F. Moftakharzadeh, Montogomery identities for fractional integrals and related fractional inequalities, J. Ineq. Pure Appl. Math. 10(4) (2009), Article ID: 97.

[2] S. Belarbi and Z. Dahmani, On some new fractional integral inequalities, J. Ineq. Pure Appl. Math. 10(3) (2009), Article ID: 86.

[3] Z. Dahmani, New inequalities in fractional integrals, International Journal of Nonlinear Scinece, 9(4) (2010), 493-497.

[4] Z. Dahmani, On Minkowski and Hermite-Hadamard integral inequalities via fractional integration, Ann. Funct. Anal. 1(1) (2010), 51-58.

[5] Z. Dahmani, L. Tabharit and S. Taf, New generalizations of Gruss inequality using RiemannLiouville fractional integrals, Bull. Math. Anal. Appl. 2(3) (2010), 93-99.

[6] Z. Dahmani, L. Tabharit and S. Taf, Some fractional integral inequalities, Nonl. Sci. Lett. A $\mathbf{1}(2)(2010), 155-160$.

[7] S. S. Dragomir and C. E. M. Pearce, Selected Topics on Hermite-Hadamard Inequalities and Applications, RGMIA Monographs, Victoria University, 2000.

[8] S. S. Dragomir, M. I. Bhatti, M. Iqbal and M. Muddassar, Some new fractional integral HermiteHadamard type inequalities, J. Comput. Anal. Appl. 18(4) 655-661.

[9] R. Gorenflo and F. Mainardi, Fractional Calculus: Integral and Differential Equations of Fractional Order, Springer Verlag, Wien, 1997.

[10] M. Iqbal, S. Qaisar and M. Muddassar, A short note on integral inequality of type HermiteHadamard through convexity, J. Comput. Anal. Appl. 21(5) 946-953.

[11] M. I. Bahtti, M. Iqbal and S. S. Dragomir, Some new fractional integral inequalities HermiteHadamard type inequalities, J. Comput. Anal. Appl. 16(4) 643-653.

[12] M. E. Özdemir, C. Yıldız, A. O. Akdemir and E. Set, On some inequalities for s-convex functions and applications, J. Ineq. \& Appl. (2013), Article ID: 333.

[13] S. Qaisar, M. Iqbal and M. Muddassar, New Hermite-Hadamard's inequalities for preinvex function via fractional integrals, J. Comput. Anal. Appl. 20(7) 1318-1328.

[14] M. Z. Sarikaya, E. Set, H. Yaldiz and N. Basak, Hermite-Hadamard's inequalities for fractional integrals and related fractional inequalities, Mathematical and Computer Modelling 57(9-10) (2013), 2403-2407.

[15] M. Z. Sarikaya and N. Aktan, On the generalization of some integral inequalities and their applications, Mathematical and Computer Modelling 54(9) (2011), 2175-2182.

\author{
${ }^{1}$ Department of Mathematics \\ Comsats Institute of Information TeChnology \\ SAHIWAL, PAKISTAN \\ E-mail address: shahidqaisar90@ciitsahiwal.edu.pk
}




\author{
2 Department of Mathematics \\ University OF ENGINEERING AND TECHNOLOGY \\ LAHORE, PAKISTAN \\ E-mail address: iqbal_uet68@yahoo.com \\ ${ }^{3}$ Department of Mathematics \\ Govt. Islamia College Civil Line \\ LAHORE, PAKISTAN \\ ${ }^{4}$ Department of Mathematics \\ College of Science, QAssim University \\ P.O. Box 6644, Buraydah 51482, Saudi Arabia \\ E-mail address: sabiriub@yahoo.com \\ ${ }^{5}$ Department of Mathematics \\ Comsats Institute of Information TeChnology \\ LAHORE, PAKISTAN \\ E-mail address: saadihsanbutt@gmail.com
}

